\title{
Studyof Crosseddipole Antennafor UHF SatelliteCommunication Applicationsand its limitations
}

\author{
Karedla Chitambara Rao ${ }^{1}$, PrudhiviMallikarjuna Rao ${ }^{2}$
}

\begin{abstract}
$\overline{\text { Abstract: In the present day communication systems, especially for Submarine applications, a compact antenna }}$ is needed to communicate with Satellites. For communicating with Satellites, the compact antenna must have circular polarization characteristics, positive gain at $\pm 45^{\circ}$ points of the main beam and the main beam position has to be in vertical direction. In this paper, the crossed dipole antenna is proposed, studied its characteristics and limitations for the ultra-high frequency Satellite Communication applications. Moreover the proposed antenna will be simulated and analyzed for various characteristics such as VSWR, Gain and Axial ratio and Radiation patterns at the downlink and uplink frequencies of the ultra-high frequency Satellite Communication applications.
\end{abstract}

Index Terms:Dipole Antenna, Crossed Dipole Antenna, UHF SatelliteCommunication.

\section{Introduction}

A dipole antenna is most commonly a linear metallic wire or rod with a feed point at the entre. Dipole antennas and arrays of dipoles are commonly used for HF and UHF Communication, TV and FM broad casting and as electric field probes. The EM field radiated by antennas has both a magnitude and a direction

Drawbacks of theDipole antenna: Most of the energy radiated from dipole or bow - tie antennas is linearly polarized and oriented predominantly along the long axis of the antenna. A complete polarization mismatch using dipole antennas results when the scattered field and polarization of the receiving antennaare both linearly polarized and oriented at right angles to each other.

- The radiation resistance of an antenna that emits dipole radiation is proportional to the square of the peak (electric or magnetic) dipole moment of the antenna. This dipole moment is roughly the product of the peak charge times the length of the antenna in the case of a timer (electric) antenna and is the product of the peak current times the area of the antenna in the case of a loop (magnetic) antenna. Hence it is hard to increase the radiation resistance of small linear or loop antennas by altering their shapes.

- In the near zone the magnetic field B is in phase with the current in a simple small antenna, while the electric field $\mathrm{E}$ is in phase with the charge but the charge and current have a $90^{\circ}$ phase difference. Hence they imply the electric and magnetic fields are $90^{\circ}$ out of phase in the near zone, so that the radiation (which is proportional to EXB) is weak.

\section{Crossed - dipole antenna:}

The crossed - dipole antenna consists of transmit and receive antennas oriented orthogonal to each other and is sensitive to field components oriented parallel to the long axis of the receive antenna. So far many authors were studied and simulated the crossed dipole antenna for different applications. No one is simulated the crossed dipole antenna for UHF satellite communication application and analyzed the various characteristics like VSWR, Gain and axial ratio. The authors presented the characteristics, designs and applications of crossed dipole antenna [1] and they had discussed why dual - feed crossed dipoles have been installed in many broadband antennas. They said that the dual feed crossed dipole antennas can be designed to radiate isotropic, omnidirectional, unidirectional $\mathrm{CP}$ and unidirectional DP radiation. They described how $\mathrm{CP}$ radiation can be obtained with a single - feed structure by using the exact lengths of orthogonal dipoles. They also reviewed the recent including bandwidth enhancement, multiband operation and radiation central. They illustrated the different techniques to achieve multiple resonance and compact size. They also described compact radiators with different reflectors like cavity backed, AMC surface and planar metallic to achieve broadband and unidirectional radiation patterns. They discussed the importance of crossed dipole antenna [2] when it was used in high noise environments. The signal to noise ratio can be improved by using crossed dipole antenna. Reduction of cross- coupling of crossed dipole can produce cleaner images under suitable conditions and depending on the targets of interest. A method was proposed [3] to broaden the beam width of a crossed dipole antenna. By using four parasitic strips around the crossed dipole antenna, the beam widths were broadened effectively in the vertical plane over a wide frequency band. An L-band prototype $(1.1 \mathrm{GHz}$ to $1.6 \mathrm{GHz}) \mathrm{crossed}$ dipole antenna was fabricated and tested. The beam width at lower frequencies was broadened and uniform radiation patterns over the whole operating frequency band.A single feed wide band circularly polarized antenna was proposed for GNSS. The authors was used [4] the two orthogonal bowtie dipoles with double printed 
quarters rings for getting matching with a antenna to a single $50 \Omega$ co- axial line to produce $\mathrm{CP}$ radiation. The prototype antenna was designed and tested with an overall $1.2 \mathrm{GHz}$ frequency size of $0.48 \lambda_{0} \mathrm{x}$ $0.48 \lambda_{0} \times 0.25 \lambda_{0}$. They had measured a value of $\left|S_{11}\right|<-10 \mathrm{~dB}$ band width of $1.05-1.79 \mathrm{GHz}$ and also measured a3$\mathrm{dB}$ bandwidth of $1.12-1.64 \mathrm{GHz}$. Finally they got the RHCP with a small gain variation and high radiation efficiency $(>93 \%)$ over the operational bandwidth. The wideband dual - polarized crossed - dipole antenna with the parasitical crossed strip [5] was presented and investigated. The parasitical crossed strips were used to achieve another resonant point and improved impedance band width. They had measured the values of 34.9\% for $S_{11} \leq-10 \mathrm{~dB}\left(+45^{0}\right.$ Polarization) and $S_{22} \leq-10 \mathrm{~dB}\left(-45^{0}\right.$ Polarization). A compact [6] dual band antenna was presented for WLAN applications. This antenna consists of a dual band HIS and a cross - dipole. The simulated results of compact dual band indicates that gain of $3.8 \mathrm{dBi}$ at $\mathrm{f}_{\text {low }}=2.4 \mathrm{GHz}$ and $6.3 \mathrm{dBi}$ at $\mathrm{f}_{\text {high }}=5.4 \mathrm{GHz}$ in $\mathrm{z}$ direction.A broadband printed cross - dipole element and feed network were designed [7] and fabricated to test four polarization re - configurations. The four polarizations are two linear and two circular in the operating band $1.7-2.5 \mathrm{GHz}$. The broadband polarization reconfiguration were implemented using broadband $90^{\circ} / 180^{\circ}$ phase shifters of switched network types. The fabricated BPCDE - PR showed the good performance in the operating band of about 40\%.A dual band short backfire antenna was [8] developed based on the cross dipole excitation structure single impedance band width characteristics. The cross dipole excitation structure consists of $\mathrm{H}$-shaped and straight cross dipoles. This cross dipole structure was mounted on the slotted co-axial time and developed on elliptical ground plane with dielectric arm. A cost effective design was obtained by using metallic rim from dielectric rim. They achieved the antenna characteristics such as VSWR bandwidth of $21 \%$ and $20.36 \%$ for $2: 1$ VSWR, axial ratio of $1.8 \mathrm{~dB}$ with axial ratio $(\leq 3 \mathrm{~dB})$ bandwidth of $1.94 \%$ and a gain of $12 \mathrm{dBi}$. A novel circularly polarized antenna was [9] presented for broadband UHF Satellite Communication applications based on Moxon type antenna (bent dipole element over a ground plane). The broadband operation was achieved by using two vertical elements of the Moxon arms, widened strip arm element, bow tie arm structures with bents at $90^{\circ}$. The presented antenna was fabricated and measured the results for the band of $225-400 \mathrm{MHz}$. the measured results are gain of $8-12 \mathrm{~dB}, \mathrm{~S}_{11}$ is $10 \mathrm{~dB}$. An array of sequentially crossed dipole antenna [10] was designed to get excellent circular polarization and input impedance characteristics. A loaded crossed dipole antenna with broad - band omnidirectional horizontally polarized [11] was designed based on the two steps. First one was best omnidirectional pattern in the horizontal plane and second was antenna with the best performance was loaded with resonant tank circuits and a matching network. Finally a QA was used to determine the load components, their locations and the parameters of the matching network.

Based on low profile cross - dipole and EBG/AMC structure, a new 8- port [12]interleaved base station antenna was designed. Three adaptive orthogonal crossed- dipoles [13] were used to remove the losses due to directivity and polarization mismatch if tracked by the transmit antenna. A GA quickly adapts the receive antenna to transmit antenna. Two antennas were designed [14] such as crossed meander antenna and crossed staircase antenna for RFID based sensor networks. Both antennas were designed to operate in the unlicensed $915 \mathrm{MHz}$ frequency band. A novel small size multi - band cross dipole antenna [15] was designed at the frequencies $0.543 \mathrm{GHz}, 2 \mathrm{GHz}$ and $6.5 \mathrm{GHz}$ based on a fractal first iteration quadraticand triangular Koch curve. The Method of Moments (MOM) techniques was used to calculated the current distribution along the cross fractal Koch curve antenna. A new compact dual polarization antenna was [16] designed at $2.23 \mathrm{GHz}$ and $5.14 \mathrm{GHz}$. The dual polarized antenna consists of a rectangular patch with notches combined by a crossed dipole in the same ground and substrate by optimized of this new structure. The circularly polarized crossed Dipole Antenna was [17] designed based on the unequal length dipoles and two metal strips with phase delay lines for RFID hand held reader. They had measured the impedance bandwidth (VSWR $\leq 2$ ) of about $11.8 \%$ and a 3 -dB axial - ratio (AR) bandwidth of about $3.3 \%$ around the center frequency of $925 \mathrm{MHz}$. The symmetric crossed dipole FSR was [18] designed based on the frequency selection reflectarray for wireless communication applications. The FSR has the ability to function as a reflector and steer reflected beam for a special frequency band wave. The non - symmetric FSR using non - symmetric crossed dipoles antenna was also designed for dual - source and dual polarization operation with two main beams simultaneously. Moreover the FSR was implemented in a WCDNA system to eliminate the blind spots in Wireless Mobile Communications.

\section{CROSSED DIPOLE MODEL}

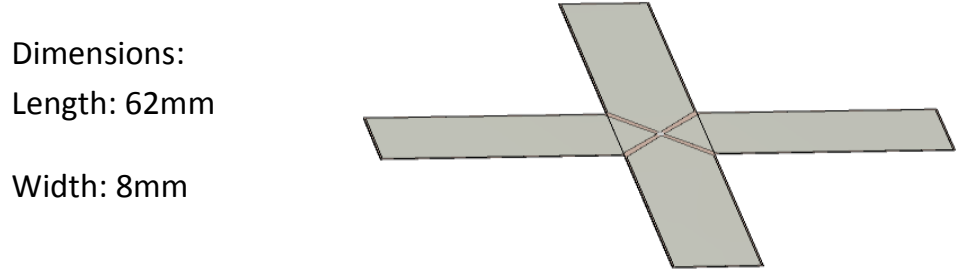

Fig. 1.Crossed dipole antenna. 
In the crossed dipole antenna, two half wave dipoles placed at right angles to each other in the same plane, excited $90^{\circ}$ out of phase with each other. The dipoles carry equal current in magnitude but in phase quadrature. The crossed - dipole arrangement is shown in fig.2 along with the directional pattern of individual and of the combination. Here two dipole patterns combining in the manner shown in figure, producing an almost circular pattern in the plane of crossed - dipole. The pattern also has the feature that it is polarized in the same plane. So that of it were mounted in the horizontal plane the antenna would radiate horizontally polarized wave about equally well in all directions along the ground.

2



$\mathrm{E}_{2}=k \cos \theta$

0



$\mathrm{E}_{1}=\mathrm{k} \sin \theta$

Fig.2 (a) plane view of Crossed diploe antenna Fig.2 (b) Vector diagram of field at a point P Fig. 2

Fig.2 (a) shows plan view of two short crossed dipoles. At a distant point $\mathrm{P}$ in the direction $\theta$ to the dipole no.1, the field magnitude due to dipole no. 1 is proportional to $\sin \theta$ and that due to dipole no. 2 is proportional to $\cos \theta$. The vectors representing these two fields are however at $90^{\circ}$ time phase to one another. Hence the resultant field at point ' $\mathrm{P}$ ' is the vector sum of two vectors at right angles to one another and proportional to $\sin \theta \operatorname{and} \cos \theta$ as illustrated in fig. 2 (b). The resultant is, therefore, a constant independent of $\theta$. If the short dipoles are replaced by a half wave dipoles the change in the pattern in a horizontal plane is not very great and this forms the basis of the crossed dipole antenna. If the field on a vertical axis above the antenna is examined, it is found that the electric vector rotates in a horizontal plane Fig.3 (a). The instantaneous field straight in a horizontal plane due to dipole no. 1 is saying proportional to sin $\omega$ t and in the direction OX. The instantaneous field due to dipole no. 2 is


circular pattern in the $\theta$ plane is given by

$$
\begin{array}{r}
E=\sin \omega t \cos \theta+\cos \omega t \sin \theta \\
E=\operatorname{Sin}(\omega t+\theta)(2) \\
|E|=1(3)
\end{array}
$$

Thus at any value of $\theta$, the maximum amplitude of $E$ is unity at some instant during each cycle. The rms filed pattern is circular Fig.3(c). At instant of time the pattern is figure of eight of the same shape as for a single infinitesimal dipole. The instantaneous patterns are shown in Fig.3(c) The resultant filed is a vector of fixed amplitude rotating in a horizontal plane about a fixed axis. Reversal of the connections to one dipole reverses the direction of rotation.

\section{Simulation results}

According to the simulation results, it has been observed that from Fig.3, the VSWR value is high at the low frequencies and it will goes on decrease as the frequency increases from the $150 \mathrm{MHz}$ to $200 \mathrm{MHz}$. Again the VSWR value increases as the frequency increases from $200 \mathrm{MHz}$ to $400 \mathrm{MHz}$.Fig.4shows plot of gain versus frequency. From this plot it has been observed that the gain value is more negative below $200 \mathrm{MHz}$, after $200 \mathrm{MHz}$ the gain value is almost constant and less negative up to $400 \mathrm{MHz}$. Fig.5.shows that Radiation pattern characteristics of Crossed dipole antenna at downlink and uplink frequencies of the UHF Satellite Communication at phase angle $0^{\circ}, 180^{\circ}, 90^{\circ}$ and $270 .{ }^{\circ}$ From the Radiation pattern characteristics it has been observed that there is no radiation in the vertical direction. Table I and TableII shows that main lobe magnitude, main lobe direction and angular width at the downlink and uplink frequencies of the UHF Satellite Communication. From Table1 and Table2 it has been observed that main lobe magnitudes are negative values around $-2 \mathrm{~dB}$. 




Fig. 3.VSWR ofUHF Crossed dipole antenna.

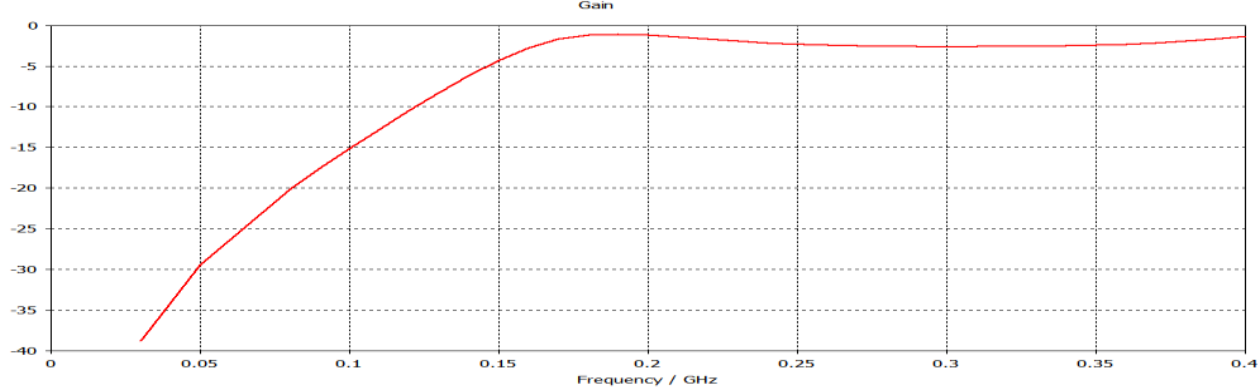

Fig. 4.Gain of UHFCrossed dipole antenna

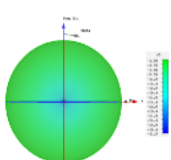

LHCP @ $240 \mathrm{MHz}$



LHCP@250 MHz



CUT PLANE@240 MHz
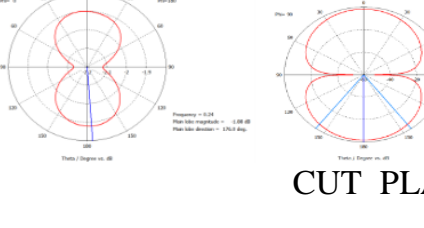

$\ldots$



CUT PLANE @250MHz





LHCP @270 MHz



CUT PLANE @270MHz 




LHCP @300 MHz



LHCP @310 MHz



LHCP @320 MHz

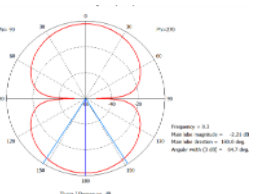

CUT PLANE@300 MHz


CUT PLANE @320 MHz

Fig. 5. Radiation patterns of UHF Crossed dipole antenna at various operating frequencies.

Table I

Main lobe magnitude, Main lobe directionatphase angle $0^{0} \& 180^{\circ}$ for various frequencies

\begin{tabular}{|c|c|c|}
\hline $\begin{array}{c}\text { Frequency } \\
(\mathrm{MHz})\end{array}$ & $\begin{array}{c}\text { Main lobe Magnitude } \\
(\mathrm{dB})\end{array}$ & $\begin{array}{c}\text { Main lobe direction } \\
\text { (Degrees) }\end{array}$ \\
\hline $240 \mathrm{MHz}$ & -1.88 & 176.0 \\
\hline $250 \mathrm{MHz}$ & -2.04 & 174.0 \\
\hline $260 \mathrm{MHz}$ & -2.16 & 172.0 \\
\hline $270 \mathrm{MHz}$ & -2.24 & 170.0 \\
\hline $290 \mathrm{MHz}$ & -2.26 & 173.0 \\
\hline $300 \mathrm{MHz}$ & -2.21 & 176.0 \\
\hline $310 \mathrm{MHz}$ & -2.14 & 178.0 \\
\hline $320 \mathrm{MHz}$ & -2.04 & 179.0 \\
\hline
\end{tabular}

Table II

Main lobe magnitude, Main lobe directionat phase angle $90^{\circ} \& 270^{\circ}$ for various frequencies

\begin{tabular}{|c|c|c|c|}
\hline $\begin{array}{c}\text { Frequency } \\
(\mathrm{MHz})\end{array}$ & $\begin{array}{c}\text { Main lobe } \\
\text { Magnitude } \\
(\mathrm{dB})\end{array}$ & $\begin{array}{c}\text { Main lobe } \\
\text { direction } \\
\text { (Degrees) }\end{array}$ & $\begin{array}{c}\text { Angular } \\
\text { width } \\
(3 \mathrm{~dB})\end{array}$ \\
\hline $240 \mathrm{MHz}$ & -1.88 & 180.0 & 74.2 \\
\hline $250 \mathrm{MHz}$ & -2.05 & 180.0 & 72.8 \\
\hline $260 \mathrm{MHz}$ & -2.17 & 180.0 & 71.4 \\
\hline $270 \mathrm{MHz}$ & -2.24 & 180.0 & 69.9 \\
\hline $290 \mathrm{MHz}$ & -2.26 & 180.0 & 66.5 \\
\hline $300 \mathrm{MHz}$ & -2.21 & 180.0 & 64.7 \\
\hline $310 \mathrm{MHz}$ & -2.24 & 180.0 & 62.9 \\
\hline $320 \mathrm{MHz}$ & -2.06 & 180.0 & 60.5 \\
\hline
\end{tabular}

\section{Limitations of crossed dipole antenna}

- From the simulation results such as gain and radiation pattern characteristics, it has been observed that gain and main lobe magnitude are negative values. For communicating with satellites the antenna gain as well as main lobe magnitudes both are must be positive then only the antenna can track the satellite directly if not ,the antenna cannot tracks the satellite. This is the main limitation of the crossed dipole antenna when it is used for UHF Satellite Communication applications.

- There is another limitation which is VSWR. For UHF Satellite Communication applications, the VSWR value should be low as possible and VSWR $\leq 3$ is accepted. From the VSWR versus frequency characteristics, it has been observed that VSWR value is high value at the frequency range from $100 \mathrm{MHz}$ to $400 \mathrm{MHz}$. This high value of VSWR is not appropriate for UHF Satellite Communication applications. 


\section{Conclusion and future work}

It is concluded that the Crossed dipole antenna has been studied its characteristics and limitations for UHF Satellite Communication applications. The Crossed dipole antenna was simulated and analyzed for various characteristics such as VSWR, Gain and Radiation pattern characteristics at the downlink and uplink frequencies of the UHF Satellite Communication. From the simulation results, it has been observed that the Crossed dipole antenna cannot be used for communicating with Satellites because the gain value is negative and it is around $2 \mathrm{~dB}$ at the downlink and uplink frequencies .Apart from this, the main lobe magnitude values are also negative they are around $-2.01 \mathrm{~dB}$ from $240 \mathrm{MHz}$ to $320 \mathrm{MHz}$. Moreover VSWR value is high and not accepted for UHF Satellite Communication. Bifilar helix antenna is the suitable antenna for communicating with Satellites because helix antenna has unique characteristics such as circular polarization, wide axial ratio and wide beam width. Our future work will bethe study, design, simulation, fabrication and testing of a bifilar helix antenna for various Communications like UHF, GPS, GLONASS, IRNSS and S-Band

\section{References}

[1]. Son Xuat Ta, Lkmo park and Richard co. Ziolkowski "Crossed Dipole Antennas: A review", IEEE Antennas \& Propagation Magazine, October 2015

[2]. Stanley J. Radzevicius, Jeffrey J. Daniels "Significance of crossed dipole antennas for high noise environments" proceedings of the symposium on the Application of Geophysics to Environmental and Engineering Problems, 2000, the Environmental and Engineering Geophysical Society, PP:407-413.

[3]. Y.-F.Wei, B.-H. Sun, “ Method for Broadening the beam widths of Crossed dipoles for wideband GPS applications”, Progress in Electromagnetic Research letters,Vol.12, 31-40, 2009

[4]. Hug Hung Tran. Son XuatTa. Ikmoparle"Single Feed, Wideband, Circularly polarized, Crossed Bowtie Dipole Antenna for Global Navigation Satellite Systems" Journal of Electromagnetic Engineering and Science, Vol. 14, No.3, September 2014

[5]. Shao - Li Zuo, Qiong - Qiong Liu, and Zhi - ya Zhang “ Wideband Dual - Polarized Crossed - Dipole Antenna with parasitical Crossed - Strip for Base Station Applications “ Progress In Electromagnetic Research C, Vol.48, 159 - 166, 2014.

[6]. D. Kornek, S. Hampel“Performance of printed Dipole on Dual Band High - Impedance Surface", 2009 International ITG Workshop on smart Antennas -WSA 2009, February 16-18, Berlin, Germany.

[7]. Soon - Young Eom, IC - Pyo Hong and Jong - Myeon Kim "Broadband Printed Cross - Dipole Element with Four polarization Reconfigurations for mobile Base station Array Antenna Applications" Hindawi Publishing Corporation, International Journal of Antennas and Propagation, volume 2011.

[8]. M. JavidAsadand M. Zafrullah "Cost Effective Dual Band Short Backfire Antenna" International Journals of Electrical \& Computing Sciences IJECS - IJENS, vol.9, No:9

[9]. Ibrahim Takin, Oksana Manzhura and EdipNiver"Broadband Circularly Polarized antennas for UHF SATCOM".

[10]. J.C.Bregains "Circularly Polarized Arrays of Sequentially crossed dipoles" Journal of current Engineering Research, Volume 3, Issue 6, Nov-Dec 2013.

[11]. KarimMohammad pour - Aghdam"Miniaturized Loaded Crossed Dipole Antenna with Omni - Directional Radiation pattern in the Horizontal plane" proceedings of the $39^{\text {th }}$ European microwave Conference, October 2009.

[12]. Fayez Hyjazie, pave Watson and HalimBoutayeb"Dual band International Base Station Phased array antenna with optimized cross dipole and EBG/AMC Structure".

[13]. Randy L. Haupt "Adaptive Crossed Dipole Antennas using a Genetic Algorithm”.

[14]. R.H. Bhuiyan,R.Dougal and M. Ali "A New Crossed Staircase Dipole Antenna for 915MHz RFID Application".

[15]. Fawwaz Jinan Jibrael “ Multiband cross Dipole Antenna Based on the Triangular and Quadratic Fractal Koch curve” International Journal of Engineering (IJE), vol.4, Issue 3.

[16]. ZynebBerkat and NovreddineBoukliHaccne "Design of New combine Antenna for Dual Polarization using crossed Dipole at 2.23GHz and 5GHz" ARPN Journal of Engineering and Applied sciences, Vol.10, No.12, July 2015.

[17]. Yi- Fang Lin, Yang - Kai Wang "Circularly polarized Crossed Dipole antenna with Phase Delay lines for RFID Handheld Reader" IEEE Transactions on Antennas and Propagation, Vol.60, No.3, March 2012.

[18]. Long Li,Qiang Chen "Frequency SelectiveReflectarray using Crossed - Dipole elements with Square loops for wireless Applications" IEEE Transactions on Antennas and Propagation, Vol.59, No.1, January 2011. 